\title{
The Adult Visual Cortex Expresses Dynamic Synaptic Plasticity That Is Driven by the Light/Dark Cycle
}

\author{
Marian Tsanov and Denise Manahan-Vaughan \\ Learning and Memory Research, Medical Faculty, and International Graduate School for Neuroscience, Ruhr University, 44780 Bochum, Germany
}

\begin{abstract}
Experimental evidence derived from in vitro studies suggests that synaptic plasticity may be involved in information processing in the adult sensory cortex. Here, we demonstrate, for the first time, that synaptic plasticity that endures for $>24 \mathrm{~h}$ can be elicited in the visual cortex of freely moving adult rats. Both long-term potentiation (LTP) and long-term depression (LTD) were evoked in layer 2/3 of the primary visual cortex after stimulation of the dorsal lateral geniculate nucleus. We found that synaptic plasticity within visual cortex synapses is not a static phenomenon, where a particular pattern of stimulation invariably evokes the same form of synaptic plasticity but rather fluctuates in association with diurnal luminance levels. Whereas acute $(12 \mathrm{~h})$ dark exposure drives intrinsic synaptic transmission to basal levels, light exposure $(12 \mathrm{~h}$ ) leads to synaptic potentiation. Furthermore, low-frequency stimulation at $3 \mathrm{~Hz}$ generates LTD in light-exposed animals, and LTP in dark-exposed animals, in close correlation with the time course of natural slow wave oscillations. Our data demonstrate that the adult visual cortex is in a perpetually dynamic state, where the direction of plasticity changes depends on the immediate visual experience.
\end{abstract}

Key words: lateral geniculate nucleus; layer 2/3; thalamocortical synapse; synaptic plasticity; in vivo; LTP

\section{Introduction}

In the visual cortex (VC), synaptic plasticity contributes to experience-dependent acquisition of visual responsiveness during the critical period (Bear et al., 1987; Singer, 1995). Thus, in light-deprived postnatal rats, long-term potentiation (LTP) is enhanced, and long-term depression (LTD) is diminished, over a range of stimulation frequencies, both in vitro and in anesthetized animals (Heynen et al., 1996; Kirkwood et al., 1996). A functional consequence of this predisposition toward LTP is an enhanced cortical response to visual stimuli, even in adult animals, suggesting a greater potential for modification of synaptic efficacies in the adult visual cortex than previously appreciated (Heynen and Bear, 2001). Studies, in vitro, demonstrated that prolonged light deprivation (over a period of weeks) alters the properties of synaptic plasticity by shifting the frequency-response function to the left (Kirkwood et al., 1996). Very little is known about the physiological conditions that drive LTP or LTD in the visual cortex of intact behaving adult animals.

Light deprivation over days and weeks does not usually occur in nature. This provokes the question as to what extent light exposure drives synaptic weight change under naturalistic circumstances. The main goal of the present study was therefore to examine visual cortex plasticity in freely moving rats and describe its relationship to diurnal changes in luminance. Do acute

\footnotetext{
Received Dec. 11, 2006; revised June 1, 2007; accepted June 17, 2007.

This work was supported by Deutsche Forschungsgemeinschaft Grant SFB509/TP C13 (D.M.-V.). We are grateful to Jens Klausnitzer and Dr. Arthur Bikbaev for technical assistance and advice.

Correspondence should be addressed to Dr. Denise Manahan-Vaughan, Learning and Memory Research, Medical Faculty, Ruhr University, Forum Nord 0st 1/116, Universitaetsstrasse 150, 44780 Bochum, Germany. E-mail: dmv-igsn@rub.de.

DOI:10.1523/JNEUROSCI.1101-07.2007

Copyright $\odot 2007$ Society for Neuroscience $\quad$ 0270-6474/07/278414-08\$15.00/0
}

changes in luminance levels, as occur in a normal diurnal cycle, have an effect on intrinsic excitability and synaptic plasticity in the visual cortex? Experience-dependent synaptic plasticity derives not just as a consequence of a modified synaptic response but rather as a result of the expression of multiple synaptic properties within a network (Morris, 1990). An adequate circuit-level description of information processing within the visual cortex is essential to bridge the gap between synapse and behavior and to understand the relationship between synaptic plasticity and information processing in the visual cortex. Our data show, for the first time, that diurnal variations in luminance trigger prolonged changes in the field response, which are in turn coupled to circadian phases. This strongly influences the ability of the animal to generate synaptic plasticity depending on the degree of thalamocortical arousal. In agreement with our data, intracellular cortical recordings in anesthetized animals have demonstrated previously an augmentation of neuronal responsiveness resulting from slow-wave sleep (SWS) oscillations (Steriade et al., 1998). This may be associated with consolidation of acquired synaptic alterations (Timofeev et al., 2000; Steriade and Timofeev, 2003). We thus also addressed the possibility that slow-frequency rhythms may influence the basal tonus of synaptic transmission in visual cortex over a $24 \mathrm{~h}$ period. We observed that low-frequency stimulation (LFS) at $3 \mathrm{~Hz}$ generates LTD in light-exposed animals, and LTP in dark-exposed animals, in close correlation with the time course of natural slow wave oscillations. Our data support that the adult visual cortex is in a perpetually dynamic state, where the direction of change of synaptic strength tightly depends on the immediate visual experience of the animal.

\section{Materials and Methods}

Surgical preparation. Under sodium pentobarbitone anesthesia (Nembutal; 40 mg/kg, i.p.), 7- to 9-week-old male Long-Evans rats (Charles River 
Breeding Laboratories, Sulzfeld, Germany) underwent implantation of a monopolar recording and a bipolar stimulating electrode (made from 0.1 -mm-diameter Teflon-coated stainless-steel wire) in the primary visual cortex and dorsolateral geniculate nucleus (dLGN), respectively.

For the recording electrode, a drill hole was made ( $1 \mathrm{~mm}$ in diameter), 7.1-7.3 $\mathrm{mm}$ posterior to bregma and 3.4-3.6 $\mathrm{mm}$ lateral to the midline, corresponding to the primary visual cortex in the rat (Zilles, 1985). A second drill hole ( $3.8 \mathrm{~mm}$ posterior to bregma, $3.3-3.4 \mathrm{~mm}$ lateral to midline) was made for a bipolar stimulating electrode. The dura was pierced through both holes using a needle. A recording electrode was lowered $200-250 \mu \mathrm{m}$ from the dural surface to reach the supragranular layer $2 / 3$. A stimulating electrode was positioned in the dLGN, ipsilateral to the hemisphere from which visual cortex recordings were obtained. The depth was 3.7-4.7 $\mathrm{mm}$ from the dural surface, corresponding with the position of the anterior pole where optic radiation fibers are bundled in close proximity (Hughes, 1977; Molnar et al., 1998). Final positions of the stimulating and recording electrodes were then determined by maximizing the amplitude of the field potential (FP) recorded in the visual cortex in response to electrical stimulation of the dLGN. Monopolar recordings from the primary visual cortex were made relative to ground and reference screws inserted into the contralateral parietal and frontal bones. For bilateral implantations, the same procedure was conducted at the other hemisphere. Once verification of the location of the electrodes was complete, the entire assembly was sealed and fixed to the skull with dental acrylic (Paladur; Heraeus Kulzer, Wehrheim, Germany). The animals were allowed $10 \mathrm{~d}$ to recover from surgery before experiments were conducted. Throughout the experiments, the animals could move freely within the recording chamber $(40 \times 40 \times 40 \mathrm{~cm})$, because the implanted electrodes were connected by a flexible cable and swivel connector to a stimulation unit and amplifier. Apart from the insertion of the connector cable at the start of the experiment, disturbance of the animals was kept to an absolute minimum. Throughout the experiments, the electroencephalogram (EEG) of each animal was continuously monitored.

Measurement of evoked potentials. The field EPSP (fEPSP) slope was used as a measure of excitatory synaptic transmission in the V1 region. To obtain these measurements, an evoked response was generated in the dLGN by stimulating at low frequency $(0.025 \mathrm{~Hz})$ with single biphasic square wave pulses of $0.1 \mathrm{~ms}$ duration per half wave, generated by a constant current isolation unit. For each time point measured during the experiments, five records of evoked responses were averaged. The fEPSP was measured as the maximal slope through the five steepest points obtained on the negative deflection of the potential. By means of inputoutput (IO) curve determination, the maximum fEPSP was found, and during experiments, all potentials used as baseline criteria were evoked at a stimulus intensity that produced $40 \%$ of this maximum $(100-400 \mu \mathrm{A})$.

The animals were maintained in a $12 \mathrm{~h}$ light/dark cycle of low $(0.1-10$ lux) and high (350-400 lux) luminance conditions. Circadian time was based on Zeitgeber time (ZT), with ZT 0 denoting light on and ZT 12 denoting light off. For the experiments that required a stimulation protocol for induction of synaptic plasticity, point " 0 " indicated the time of its application. Measurements of basal synaptic transmission (baseline) were made under low-luminance conditions during the end of the dark period of the cycle. Twenty four hours later, measurements were recorded at the same circadian time and conditions. Baseline responses were collected for 30-60 min before changing luminance conditions or application of stimulation protocols. LFS protocols comprised frequencies of $1 \mathrm{~Hz}$ (900 pulses) or $3 \mathrm{~Hz}$ (900 pulses). Theta-burst stimulation (TBS) consisted of 10 bursts at $5 \mathrm{~Hz}$, with each burst containing five pulses at $100 \mathrm{~Hz}$, given four times with a $10 \mathrm{~s}$ interval between each train of 10 bursts. LTP was alternatively induced by high-frequency tetanization at $400 \mathrm{~Hz}$ ( 10 bursts of 15 stimuli; $0.2 \mathrm{~ms}$ stimulus duration; $10 \mathrm{~s}$ interburst interval). In all cases, the stimulus amplitude was the same as that used for recordings.

Luminance of the environments was assessed using a Luxmeter (MS1300 ) with a sensitivity range of 0 to 50,000 lux. The luxmeter was placed on the floor of the recording chamber and pointed at the light source. The sensor unit possessed a photosensitive surface, which recorded the total amount of falling light.

Data analysis of electrophysiological data. The baseline fEPSP data were obtained by averaging the response to stimulation of the dLGN to obtain five sweeps at $40 \mathrm{~s}$ intervals, every $5 \mathrm{~min}$ over a period of $30 \mathrm{~min}$. Electrophysiological data were then expressed as mean percentage of baseline fEPSP reading \pm SEM (SEM). Statistical significance was estimated by using factorial ANOVA and post hoc Student's $t$ and Bonferroni's tests. Using factorial ANOVA, we estimated the effects of main factors: illuminance (two levels, high/low illuminance) and stimulation (two levels, yes/ no) on the field potential amplitude. Additionally, we estimated the effect of time on the field potential values compared with the baseline period (comprising the first six recordings/time points of each experiment). The probability level, interpreted as statistically significant, was $p<0.05$.

Data analysis of network activity. Intracortical EEG was obtained by means of recordings obtained from the supragranular layer $2 / 3$ of V1. EEG was sampled at $0.5 \mathrm{kHz}$ and stored on a hard disc for additional off-line analysis. To evaluate delta $(1-3.5 \mathrm{~Hz})$, theta $(4-10 \mathrm{~Hz})$, alpha $(10-13 \mathrm{~Hz})$, beta1 $(13.5-18 \mathrm{~Hz})$, beta2 $(18.5-30 \mathrm{~Hz})$, and gamma $(30-$ $100 \mathrm{~Hz}$ ) oscillatory activity during the course of experiment, 4-s-long epochs, $1 \mathrm{~s}$ after each test pulse, were selected. Fourier analysis of artifactfree epochs was performed with Hanning window function using "Spike2" software (Cambridge Electronic Design, Cambridge, UK). The absolute values of spectral power for each individual animal were transformed into relative ones (with mean value for baseline preinjection period taken as $100 \%$ ) that were also used for statistics. For each time point, the results of Fourier analysis of five epochs were averaged. The statistical treatment and analysis of data included the calculation of descriptive statistics (mean, SEM) and ANOVA. ANOVA was aimed to estimate the effects of luminance changes (two levels, high and low luminance) of exploratory activity (two levels, active and inactive behavioral state). For the correlation analyses, we used Pearson's coefficient of comparison.

Postmortem verification of electrode site. At the end of the study, brains were removed for histological verification of electrode localization. Brain sections $(16 \mu \mathrm{m})$ were embedded in paraffin, stained according to the Nissl method using $1 \%$ toluidine blue, and then examined using a light microscope. Brains in which an incorrect electrode localization was found were discarded from the study.

\section{Results}

Diurnal changes in illuminance generate fluctuations in basal synaptic transmission in the visual cortex

To examine basal synaptic transmission in the primary visual cortex of adult freely moving rats, animals underwent implantation of bipolar stimulating electrodes in $\mathrm{dLGN}$ and a recording electrode at the surface of the ipsilateral VC (Fig. $1 A, B$ ). FPs of layer 2/3 were recorded from naive adult Long-Evans rats. Test pulse stimulation at $0.025 \mathrm{~Hz}$ generated basal synaptic responses, which were monitored every $30 \mathrm{~min}$ over a $24 \mathrm{~h}$ time period (Fig. 1C). After the first $6 \mathrm{~h}$ under low illuminance (0.1-10 lux), the change to daylight conditions (300-500 lux) induced a gradual decrease in behavioral activity, as reflected by the animals' locomotor activity (Fig. 1C). In keeping with this strong light trigger of the rodent endogenous circadian clock (Pittendrigh and Daan, 1976; Meijer et al., 1998), the period of vigilance was followed by a period of drowsiness (Fig. 1C). Coincident with this transition, an increase $(\sim 40 \%)$ of baseline FP amplitude was observed (Fig. $1 C$ ) (ANOVA; $F=24.6 ; p<0.001 ; n=4)$. This increase correlated negatively with the animals' locomotor activity (data not shown; Pearsons, $r=-0.682 ; p<0.001 ; n=7)$. Twelve hours later, recordings under low illuminance conditions revealed that basal synaptic transmission had returned to original levels. This suggests that basal synaptic transmission in the visual cortex oscillates between depressed and elevated levels in correlation with the light experience and illuminance-driven circadian activity of the animal.

To estimate whether fluctuations in FP amplitude are an intrinsic feature of the light/dark cycle, we recorded FP responses 
on different days in the transition period between low and high illuminance conditions (Fig. $2 A)(n=4)$. When responses were compared over $4 \mathrm{~d}$, no significant differences in the oscillation of FP amplitude were seen.

\section{Slow wave oscillatory activity and field potential amplitude increase concurrently in the active state}

To examine the role of thalamocortical oscillatory activity in this phenomenon, we analyzed the EEG recorded from the visual cortex during the naturally occurring elevations of basal synaptic transmission (Fig. 2B). Low-frequency rhythmic thalamic stimulation has been shown to result in a persistent increase in depolarizing synaptic responses of neocortical neurons in intact thalamocortical loops (Steriade et al., 1998). We therefore examined whether such activity modifies FPs in the visual cortex. Coincident with the start of lightinduced exploratory activity, an increase of all cortical frequencies was observed, of which delta $(1-3.5 \mathrm{~Hz})$ and theta $(4-10$ $\mathrm{Hz}$ ) continued during FP augmentation and persisted during both states of animal activity (ANOVA; for theta, $F=22.55, p<$ $0.0001, n=6$; for delta, $F=14.80, p<$ $0.0001, n=6$ ). These responses appear to relate to the transition to the nonaroused behavioral state.

Low-frequency rhythms during SWS may serve as an important cerebral function in the consolidation of functional changes acquired during wakefulness (Steriade and Timofeev, 2003). Sleep, with oscillations within the delta frequency, is implicated in cortical plasticity evoked by monocular deprivation in the developing visual cortex (Frank et al., 2001). To examine the role of slow oscillations in the modification of visual cortex firing properties, we assessed the relationship of both FP and oscillatory changes over prolonged periods of time. For this purpose, we recorded visual cortex activity constantly for $24 \mathrm{~h}$ during a natural light/dark cycle (Fig. 4C,D). Here, we recorded visual cortex FP amplitude and EEG patterns every $15 \mathrm{~min}$ for the first $12 \mathrm{~h}$ and every $30 \mathrm{~min}$ for the next $12 \mathrm{~h}$ in the course of a natural light/dark cycle (Fig. 2D,E). Theta and delta ranges began to elevate simultaneously with gamma increases during high luminance-induced exploratory behavior. They reached $300-400 \%$ of basal levels (ANOVA; for theta, $F=64.50, p<0.0001, n=4$; for delta, $F=$ $11.08, p<0.005, n=4$ ), which continued throughout the inactive state. This period was characterized by sleep cycles and significant decrease of gamma activity $(p<0.0001 ; F=21.85 ; n=$ 4; data not shown). Our data show a parallel increase of lowfrequency oscillations and FP amplitude, proposing a possible link between these two network features (Steriade and Timofeev, 2003).

\section{Frequency dependence of LTP induction in the visual cortex of freely moving animals}

The CNS regularly shifts between aroused (preparatory) and nonaroused (consummatory) or sleeping states (Sherrington,
1897). We tested the hypothesis that changes in FP amplitude are tightly linked to arousal levels in the animals that are elicited by the light/dark cycle. We therefore examined synaptic plasticity in the visual cortex during aroused (locomotor activity higher than $20 \mathrm{~cm} / \mathrm{min}$ ) versus nonaroused (locomotor activity $<20 \mathrm{~cm} /$ $\mathrm{min}$ ) states of the animal. To elicit a nonaroused, inactive state, a group of rats was exposed to high-luminance conditions (400 lux) for a few hours before starting experiments to elicit a phase advance in the circadian cycle. These animals expressed an elevated basal synaptic transmission in response to test pulse stimulation when compared with animals that were not exposed to light during the same period (aroused) (Fig. 3A) (ANOVA; for the animals exposed to light in the subsequent night, $F=10.61$, $p<0.001, n=4$; for the animals exposed to light in the preceding night, $F=8.14, p<0.001, n=6)$. The elevation of FP amplitude seen in light-primed animals reverted during the subsequent night (darkness) to levels seen in animals that had experienced the normal dark/light cycle. This latter group conversely showed no recovery of FP elevation back to dark levels when light exposure was maintained into the subsequent night period. The magnitude of the light-induced phase advance of the circadian cycle in the late night is dependent on light intensity and on the duration of light exposure (Nelson and Takahashi, 1991; Meijer et al., 1992) and reflects a suppression of locomotor activity (Pittendrigh and Daan, 1976).

A particular subset of pyramidal neurons in the visual cortex, known as chattering cells, are involved in the generation of syn- 

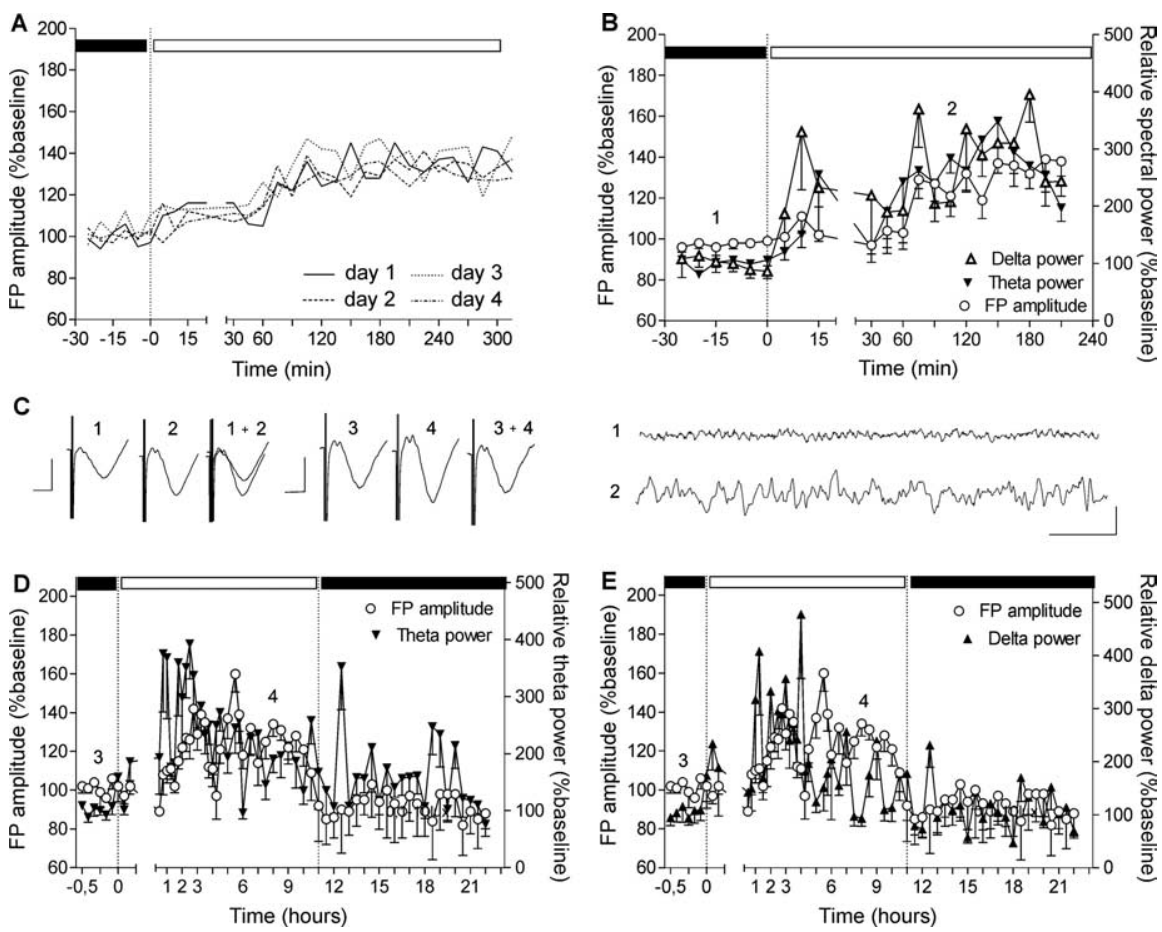

Figure 2. Slow-frequency rhythms increase concurrently with the augmentation of the basal synaptic transmission. $\boldsymbol{A}$, Baseline recordings from a group of animals $(n=4)$ conducted in different sessions. The FP augmentation has the same profile that is independent from the day of recording. For clarity of the figure, the SEs of the average means are not presented. $\boldsymbol{B}$, EEG recorded from VC during the switch between low and high illuminance of the dark/light cycle. Elevation of the slow theta and delta frequencies is preceding the FP amplitude elevation and the transition of the animal into the inactive behavioral state. $\boldsymbol{C}$, Left analogs represent FP traces evoked at the points marked in the figures. Calibration: $5 \mathrm{~ms}, 1 \mathrm{mV}$. Right analogs represent low-pass filtered EEG analog traces from epochs marked in the figures. Calibration: $1 \mathrm{~s}, 0.1 \mathrm{mV}$. Theta $(\boldsymbol{D})$ and delta $(\boldsymbol{E})$ power elevation continues during the first 3-4h of the subsequent behaviorally inactive period. After stabilization of the augmented FP amplitude, delta and theta frequencies diminish their elevation, which reaches baseline levels during the following activity state (in close association with the return of FP amplitude to basal levels).

chronous cortical oscillations and thus participate in the recruitment of large populations of cells into synchronously firing assemblies (Gray and McCormick, 1996). Chattering cells can generate bursts with intraburst frequencies of 300-750 Hz (Gray and McCormick, 1996; Steriade et al., 1998; Brumberg et al., 2000). The generation of repetitive burst discharges in these neurons is correlated with the generation of gamma frequency $(30-70 \mathrm{~Hz})$ oscillations in the local field potential (Gray and Singer, 1989). High-frequency postsynaptic bursting is also believed to accompany synaptic potentiation in adult animals (Paulsen and Sejnovski, 2000). We therefore compared the effects of applying high-frequency TBS and high-frequency stimulation protocols $(400 \mathrm{~Hz})$. Fast onset potentiation was observed only after $400 \mathrm{~Hz}$ protocols (Fig. $3 B)($ ANOVA; $F=4.01 ; p<0.05 ; n=$ $6)$. This stimulation protocol could comprise a possible mechanism for bypassing the inhibitory gate in the mature visual cortex (Rozas et al., 2001) by mimicking the fast bursting patterns of chattering cells. Although TBS-induced LTP was characterized by a slow onset, its profile was stable, and the evoked plasticity endured longer compared with $400 \mathrm{~Hz}$-induced LTP (Fig. 3C) (ANOVA; $F=4.87 ; p<0.01 ; n=5$ ). TBS induced a similar slow-onset potentiation in light-exposed and dark-exposed rats. Thus, LTP expression was not dependent on the light exposure history of the animals and their arousal state (Fig. 3C) (ANOVA; $F=0.2136 ; p>0.1 ; n=5)$. To exclude the interference of natural (circadian) augmentation on cortical FPs, we used a TBS protocol during the dark period of the light/dark cycle (ZT 18; 0.1-10 lux) while recording from both hemispheres (Fig. 3D) (ANOVA; $F=3.12 ; p<0.05$; $n=4)$. The stimulation protocol was applied only to one of the dLGN-visual cortex pathways; the other was used as a nonstimulated (i.e., non-TBS) control. Slowonset, but persistent potentiation, that lasted for at least $4 \mathrm{~h}$ occurred in response to TBS in the stimulated pathway. The contralateral, unstimulated pathway showed no comparable changes in basal synaptic transmission.

\section{Circadian-dependent activity states drive bidirectional synaptic plasticity in the visual cortex}

To address the question as to how diurnally modulated basal synaptic transmission alters the properties of synaptic plasticity in the superficial layers of visual cortex, we used a stimulation protocol known to induce LTD in the visual cortex in vitro and in anesthetized rats (Heynen et al., 1996; Kirkwood et al., 1996). We examined the relationship between synaptic plasticity response, the behavioral state, and the preceding light exposure. Two categories of behavioral state were assigned based on off-line analysis: animals from the inactive, light-exposed group were characterized by locomotor activity $<20$ $\mathrm{cm} / \mathrm{min}$; theta and delta spectral power throughout the recordings $<150 \%$, compared with baseline measurements. The rats assigned to the active, dark-exposed group were characterized by locomotor activity $>30 \mathrm{~cm} / \mathrm{min}$; theta and delta spectral power throughout the recordings $>250 \%$, compared with baseline measurements.

LFS (1 Hz; 900 pulses) generated instantaneous LTD (20$25 \%$ ) in light-exposed animals (Fig. 4A). Dark-exposed rats, which were in an aroused state after low-luminance exposure, tended toward weaker depression in response to the same protocol (Fig. 4A) $(n=4)$, suggesting a leftward shift in the LFS response function. Whether this metaplastic effect was not just quantitative but also qualitative was the next point of investigation. LFS at $3 \mathrm{~Hz}$ lies in the range of the modification threshold (Bienenstock et al., 1982; Kirkwood et al., 1996) that determines whether LTP or LTD will be generated depending on the behavioral state of the animal. Here, LFS at $3 \mathrm{~Hz}$ generated robust LTD after animal exposure to light at daylight luminance levels (Fig. $4 B)$ (ANOVA; $F=8.3 ; p<0.001 ; n=8)$. In contrast, $3 \mathrm{~Hz}$ stimulation generated robust LTP in animals kept under dark conditions for $12 \mathrm{~h}$ before LFS (ANOVA; $F=6.2 ; p<0.001 ; n=$ $7)$. The initial depression corresponded to the characteristics of activity-dependent changes in synaptic responses induced by frequencies between 3 and $10 \mathrm{~Hz}$ (Wang and Wagner, 1999). Application of $3 \mathrm{~Hz}$ stimulation, $4 \mathrm{~h}$ after the initial protocol led to short-term depression (STD) in both groups (Fig. $4 C)(n=4$ for the dark-exposed group and $n=5$ for the light-exposed group). After recovery from STD, the magnitude of LTP in the previously dark-exposed group and the magnitude of LTD in the previously light-exposed group was unchanged. This suggests that $4 \mathrm{~h}$ after 
application of LFS, synaptic plasticity is not vulnerable to interference by subsequent identical stimulation.

Thus, luminance-triggered behavioral states that correlate with the circadian cycle of the rat generated metaplasticity (Abraham and Bear, 1996) in the rat visual cortex. Concordantly, we observed a different and immediate oscillatory response in the visual cortex after $3 \mathrm{~Hz}$ stimulation of the dLGN. In dark-exposed animals that underwent potentiation of the cortical EPSP, an increase of gamma power was observed in parallel with the initial shortterm FP depression (Fig. 4D) (Bonferroni's test; $p<0.001$ ). This experiment demonstrates a relationship between gamma oscillatory activity and the metaplasticity of synaptic strength in visual cortex

\section{Discussion}

LTP and LTD may be involved intrinsically in memory processing (ManahanVaughan and Braunewell, 1999; Kemp and Manahan-Vaughan, 2004, 2005, 2007; Uzakov et al., 2005; Whitlock et al., 2006). Apart from its role in development, the visual cortex has been proposed as a memory system, involved in encoding stimulus features and in the short-term storage of sensory stimuli to guide behavior (Pasternak and Greenlee, 2005). To examine its role in information storage, we investigated whether dynamic synaptic plasticity occurs in the adult visual cortex. Our specific goal was to establish whether alterations in the diurnal light/dark cycle affect intrinsic and naturalistic plasticity in this structure. We found that long-lasting synaptic plasticity, in the form of LTP and LTD, can be elicited in the visual cortex of freely moving adult rats. Our findings support that synaptic plasticity within the adult visual cortex is not a static and stereotypic phenomenon, but rather it varies in a direct relationship with diurnal luminance levels. Whereas acute ( $12 \mathrm{~h}$ ) dark exposure drives intrinsic synaptic transmission to basal levels, light exposure (12 h) leads to synaptic potentiation. Intriguingly, the previous experience of the synapse determines the direction of synaptic plasticity elicited by afferent stimulation: low-frequency stimulation at 3 $\mathrm{Hz}$ generates LTD in light-exposed animals and LTP in darkexposed animals. These data support that the adult visual cortex is in a perpetually dynamic state, where the direction of change of synaptic plasticity depends on the immediate visual experience. These findings have substantial implications for the role of the visual cortex in information storage.

LTP and LTD are believed to underlie memory processes on a cellular level (Bliss and Collingridge, 1993; Bear, 1996; ManahanVaughan and Braunewell, 1999; Kemp and Manahan-Vaughan, 2007). However, a significant challenge comprises the precise determination of how LTP and LTD relate to naturally occurring long-term plasticity. Our study revealed that the history of cortical activity is tightly related to changes in the field EPSP response evoked by active visual processing or by thalamocortical stimulation. Neuronal responses vary as a function of network activi-
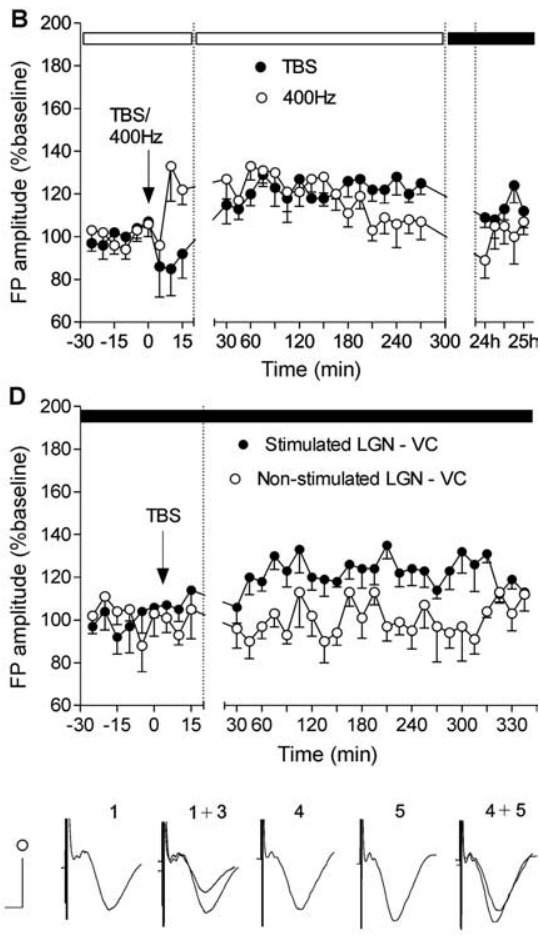

Figure 3. Long-term potentiation in visual cortex depends on the stimulation protocol but not on the activity state. $\boldsymbol{A}$, Lightendent modulation of basal synaptic transmission in the visual cortex. Rats were exposed to high-luminance for $4 \mathrm{~h}$ in the ast onset but unstable and shorter-lasting potentiation in visual cortex compared with TBS-induced slow-onset potentiation $C$ during the dark period of the natural light/dark cycle (0.1-10 lux) to exclude an interference with the diurnal FP augmentation $\left({ }^{*} p<0.05\right) . E$, Analogs represent FPs evoked at the points marked in the figures. Calibration: $5 \mathrm{~ms}, 1 \mathrm{mV}$.

ties, which depend, in turn, on behavioral states. Our results also support that basal synaptic transmission in the adult visual cortex oscillates between elevated and suppressed levels. The plasticity properties of both states differ, with LFS protocols in particular, inducing dissimilar synaptic alterations. LFS at $3 \mathrm{~Hz}$ leads to LTD in light-exposed, and LTP in dark-exposed, animals. This suggests that in the light-exposed state, the modification threshold for induction of LTD is shifted to the left (Bienenstock et al., 1982; Bear et al., 1987; Kirkwood et al., 1995), whereas a leftward shift in the modification threshold for LTP occurs in the darkexposed state. Effects occurred in close correlation with the time course of natural slow wave oscillations and are consistent with the occurrence of metaplasticity (Bear et al., 1987; Abraham and Bear, 1996) in the visual cortex in response to the phase of the day-night cycle.

We show for the first time that LTP occurs in the visual cortex of freely behaving adult animals, and that the profile of synaptic potentiation depends on the frequency of stimulation protocol. Whereas $400 \mathrm{~Hz}$ stimulation leads to immediate, yet short-lasting potentiation, TBS evokes a slow onset but persistent LTP. The time course of TBS-induced LTP parallels visual stimuli-driven and gamma oscillations-related alterations in visual cortex activity (Tsanov and Manahan-Vaughan, 2007) and confirms TBS as a model for the dynamic coupling of thalamocortical networks. Interestingly, in the hippocampus, a link between synaptic plas- 

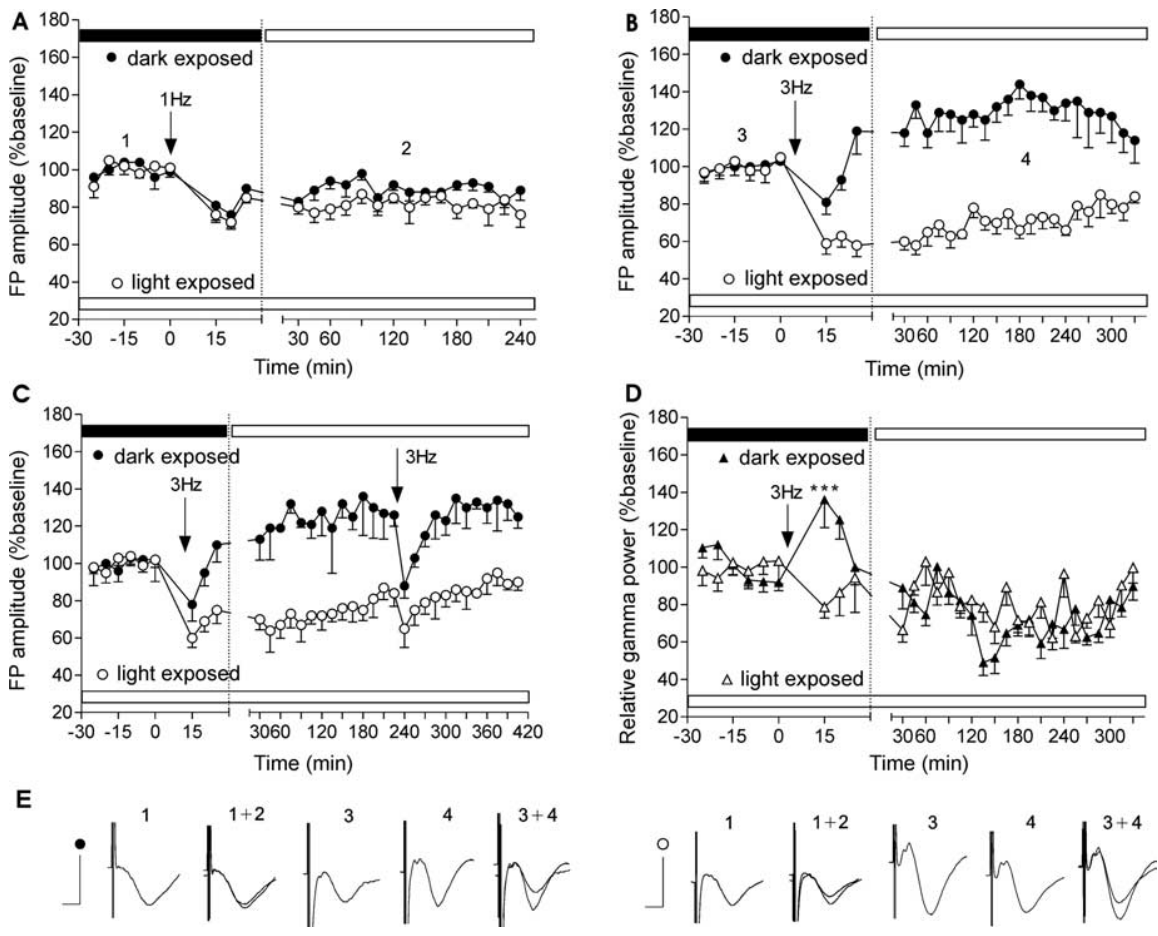

Figure 4. LFS-induced synaptic plasticity is dependent on the activity state of the animal. $\boldsymbol{A}$, Stimulation (1 Hz; 900 pulses) of dLGN induced LTD in visual cortex of animals exposed to low luminance. Light exposure for $4 \mathrm{~h}$ before commencing the experiment reduces the amplitude of subsequent LFS-induced depression. $\boldsymbol{B}$, Stimulation of $3 \mathrm{~Hz}$ ( 900 pulses) results in robust LTP when induced in low-luminance conditions but leads to robust LTD in visual cortex of rats already exposed to high-luminance conditions. C, A second application of $3 \mathrm{~Hz}$ stimulation leads to transient depression of the FP when applied $4 \mathrm{~h}$ after the $3 \mathrm{~Hz}$ initial protocol. The previously induced plasticity (LTP, LTD) recovers rapidly. D, Stimulation of $3 \mathrm{~Hz}$ ( 900 pulses) induces an immediate augmentation in gamma power in dark-exposed animals only ( $\left.{ }^{* * *} p<0.001\right)$. $\boldsymbol{E}$, Analogs represent FPs evoked at the points marked in the figures. Calibration: $5 \mathrm{~ms}, 1 \mathrm{mV}$.

ticity and physiological theta rhythms has been described (Larson et al., 1986; Buzsáki et al., 1987; Larson and Lynch, 1988). Whereas LTP is elicited by stimulation to the peak of the theta wave (Pavlides et al., 1988; Huerta and Lisman, 1995; Holscher et al., 1997), LTD occurs when stimulation is given to the trough of theta (Hyman et al., 2003).

The abovementioned findings may have substantial implications for understanding how information is processed during awake and sleep states. SWS oscillations or their experimental models have been shown to enhance the responsiveness of cortical neurons, and this effect lasts for several minutes after the end of oscillatory periods (Steriade and Timofeev, 2003). LTP induced by $3 \mathrm{~Hz}$ stimulation may thus correspond to the transition of cortical neurons into a mode of decreased responsiveness to thalamic inputs, although a bidirectional relationship exists, where corticothalamic pathways also determine the network activity of the LGN. The observed augmentation of the field responses during the inactive state is in agreement with studies showing that potentiated synapses in visual cortex neurons demonstrate a reduced ability to detect afferent synchronous thalamocortical activity (Abbott et al., 1997). The increased signal-to-noise ratio is a mechanism that acts in parallel with the hyperpolarization of thalamocortical neurons to gate information processed during sleep periods. The augmented synaptic responsiveness of neocortical neurons is a phenomenon that is not restricted to the visual cortex. The probability of monosynaptic evoked synaptic discharges in motor cortex neurons is enhanced during SWS (Steriade et al., 1974; Timofeev et al., 1996).
Therefore, similar dynamic plasticity may also occur in other thalamocortical circuits.

Our visual cortex EEG data reveal that natural increases of slow rhythmic activity (theta and delta) precede the elevation of FP amplitude in freely behaving animals. The latter event coincides with a significant drop of gamma oscillations in the visual cortex and low locomotor activity (nonarousal state). This state is dominated by SWS periods, during which changes in synaptic strength, acquired as sensory experiences, are consolidated (Timofeev et al., 2000; Steriade and Timofeev, 2003). Although, throughout SWS, thalamocortical neurons remain hyperpolarized and gate afferent signals, the neocortex possesses the intrinsic networks that are necessary to elaborate self-sustained activities (Pittendrigh and Daan, 1976). This corroborates the idea that, far from being a passive receiver, the sensory cortex plays an active role in amplifying incoming thalamic inputs (Kandel and Buzsáki, 1997). $\mathrm{Ca}^{2+}$ entry in dendrites and somata of cortical neurons occurs in SWS after slow wave oscillatory activity and may trigger long-term plastic changes (Steriade et al., 1993). The suggestion that SWS oscillations are associated with consolidation processes is supported by human studies demonstrating the role of early stages of sleep in the improvement of visual discrimination skills (Stickgold et al., 2000a,b).

Our data offer the first description of dynamic visual cortex plasticity in the mammalian brain. It is not clear, however, whether this is a phenomenon that is present in the critical period and extends throughout life. In vitro analysis supports that prolonged light deprivation during the critical period has dramatic consequences for the subsequent development of normal vision in mammals (Kirkwood and Bear, 1994; Kirkwood et al., 1995, 1996). These alterations result from changes in bidirectional plasticity that suggest that LTP and LTD cooperate in the refinement of thalamocortical synapses in early development. However, the effects on synaptic plasticity of acute light and dark exposure have not been studied in postnatal animals. Although synaptic plasticity occurring during the critical period and plasticity in the adult visual cortex share common mechanisms, they differ in certain key aspects: for example, slow structural reorganization underlies consolidation of plasticity in the critical period (Hensch, 2005), and LTD is substantially less in the adult visual cortex (Frenkel and Bear, 2004). The developmental regulation of subunit kinetics of the NMDA receptor (Monyer et al., 1994; Sheng et al., 1994; Yoshimura et al., 2003), as well as the increasing role of inhibitory circuits (Fagiolini and Hensch, 2000), are also particularly important. Furthermore, not all forms of LTP or LTD that occur during postnatal development are a result of experiencedependent cortical plasticity (Hensch et al., 1998; Renger et al., 2002). This suggests that dynamic synaptic plasticity may be a specific feature of the adult visual cortex.

Our data indicate that the adult visual cortex is in a perpetually dynamic state, where the direction of change of synaptic strength 
is determined tightly by the visual experience of the animal during the light/dark cycle. We present evidence, in the rodent visual cortex, for the existence of function-dependent bidirectional plasticity. Although TBS induces long-term increases of the field response, low-frequency stimulation, at a frequency of $3 \mathrm{~Hz}$, can determine not only the amplitude but also the direction of plasticity changes, depending on the intrinsic level of diurnally modulated basal transmission. These data suggest that the initiation and stability of the thalamocortical LTP and LTD in the adult visual cortex in vivo is acutely regulated by the light/dark cycle, and that information processing in this structure thus undergoes diurnal regulation.

\section{References}

Abbott LF, Varela JA, Sen K, Nelson SB (1997) Synaptic depression and cortical gain control. Science 275:220-224.

Abraham WC, Bear MF (1996) Metaplasticity: the plasticity of synaptic plasticity. Trends Neurosci 4:126-130.

Bear MF (1996) A synaptic basis for memory storage in the cerebral cortex. Proc Natl Acad Sci USA 93:13453-13459.

Bear MF, Cooper LN, Ebner FF (1987) A physiological basis for a theory of synaptic modification. Science 237:42-48.

Bienenstock EL, Cooper LN, Munro PW (1982) Theory for the development of neuron selectivity: orientation specificity and binocular interaction in visual cortex. J Neurosci 2:32-48.

Bliss TVP, Collingridge GL (1993) A synaptic model of memory: long-term potentiation in the hippocampus. Nature 361:31-39.

Brumberg JC, Nowak LG, McCormick DA (2000) Ionic mechanisms underlying repetitive high-frequency burst firing in supragranular cortical neurons. J Neurosci 20:4829-4843.

Buzsáki G, Haas HL, Anderson EG (1987) Long-term potentiation induced by physiologically relevant stimulus patterns. Brain Res 435:331-333.

Fagiolini M, Hensch TK (2000) Inhibitory threshold for critical-period activation in primary visual cortex. Nature 404:183-186.

Frank MG, Issa NP, Stryker MP (2001) Sleep enhances plasticity in the developing visual cortex. Neuron 30:275-287.

Frenkel MY, Bear MF (2004) How monocular deprivation shifts ocular dominance in visual cortex of young mice. Neuron 44:917-923.

Gray CM, McCormick DA (1996) Chattering cells: superficial pyramidal neurons contributing to the generation of synchronous oscillations in the visual cortex. Science 274:109-113.

Gray CM, Singer W (1989) Stimulus-specific neuronal oscillations in orientation columns of cat visual cortex. Proc Natl Acad Sci USA 86:1698-1702.

Hensch TK (2005) Critical period plasticity in local cortical circuits. Nat Rev Neurosci 6:877-888.

Hensch TK, Gordon JA, Brandon EP, McKnight GS, Idzerda RL, Stryker MP (1998) Comparison of plasticity in vivo and in vitro in the developing visual cortex of normal and protein kinase A RI $\beta$-deficient mice. J Neurosci 18:2108-2117.

Heynen AJ, Bear MF (2001) Long-term potentiation of thalamocortical transmission in the adult visual cortex in vivo. J Neurosci 21:9801-9813.

Heynen AJ, Abraham WC, Bear MF (1996) Bidirectional modification of CA1 synapses in the adult hippocampus in vivo. Nature 381:163-166.

Holscher C, Anwyl R, Rowan MJ (1997) Stimulation on the positive phase of hippocampal theta rhythm induces long-term potentiation that can be depotentiated by stimulation on the negative phase in area CA1 in vivo. J Neurosci 17:6470-6477.

Huerta PT, Lisman JE (1995) Bidirectional synaptic plasticity induced by a single burst during cholinergic theta oscillation in CA1 in vitro. Neuron 15:1053-1063.

Hughes HC (1977) Anatomical and neurobehavioral investigations concerning the thalamocortical organization of the rat's visual system. J Comp Neurol 175:311-336.

Hyman JM, Wyble BP, Goyal V, Rossi CA, Hasselmo ME (2003) Stimulation in hippocampal region CA1 in behaving rats yields long-term potentiation when delivered to the peak of theta and long-term depression when delivered to the trough. J Neurosci 23:11725-11731.

Kandel A, Buzsáki G (1997) Cellular-synaptic generation of sleep spindles, spike-and-wave discharges, and evoked thalamocortical responses in the neocortex of rat. J Neurosci 17:6783-6797.

Kemp A, Manahan-Vaughan D (2004) Hippocampal long-term depression and long-term potentiation encode different aspects of novelty acquisition. Proc Natl Acad Sci USA 101:8192-8197.

Kemp A, Manahan-Vaughan D (2005) The 5-hydroxytryptamine4 receptor exhibits frequency-dependent properties in synaptic plasticity and behavioural metaplasticity in the hippocampal CA1 region in vivo. Cereb Cortex 15:1037-1043.

Kemp A, Manahan-Vaughan D (2007) Hippocampal LTD: master or minion in declarative memory processes? Trends Neurosci 30:111-118.

Kirkwood A, Bear MF (1994) Hebbian synapses in visual cortex. J Neurosci 14:1634-1645.

Kirkwood A, Lee H-K, Bear MF (1995) Co-regulation of long-term potentiation and experience-dependent plasticity in visual cortex by age and experience. Nature 375:328-331.

Kirkwood A, Rioult MG, Bear MF (1996) Experience-dependent modification of synaptic plasticity in visual cortex. Nature 381:526-528.

Larson J, Lynch G (1988) Role of $N$-methyl-D-aspartate receptors in the induction of synaptic potentiation by burst stimulation patterned after the hippocampal theta-rhythm. Brain Res 441:111-118.

Larson J, Wong D, Lynch G (1986) Patterned stimulation at the theta frequency is optimal for the induction of hippocampal long-term potentiation. Brain Res 368:347-350.

Manahan-Vaughan D, Braunewell K-H (1999) Novelty acquisition is associated with induction of hippocampal long-term depression. Proc Natl Acad Sci USA 96:8739-8744.

Meijer JH, Rusak B, Gänshirt G (1992) The relation between light induced discharge in the suprachiasmatic nucleus and phase shifts of hamster circadian rhythms. Brain Res 598:257-263.

Meijer JH, Watanabe K, Schaap J, Albus H, Detari L (1998) Light responsiveness of the suprachiasmatic nucleus: long-term multiunit and singleunit recordings in freely moving rats. J Neurosci 18:9078-9087.

Molnar Z, Adams R, Blakemore C (1998) Mechanisms underlying the early establishment of thalamocortical connections in the rat. J Neurosci 18:5723-5745.

Monyer H, Burnashev N, Laurie DJ, Sakmann B, Seeburg PH (1994) Developmental and regional expression in the rat brain and functional properties of four NMDA receptors. Neuron 12:529-540.

Morris RG (1990) Toward a representational hypothesis of the role of hippocampal synaptic plasticity in spatial and other forms of learning. Cold Spring Harb Symp Quant Biol 55:161-173.

Nelson DE, Takahashi JS (1991) Sensitivity and integration in a visual pathway for circadian entrainment in the hamster (Mesocricetus auratus) J Physiol (Lond) 439:115-145.

Pasternak T, Greenlee MW (2005) Working memory in primate sensory systems. Nat Neurosci 6:97-107.

Paulsen O, Sejnovski TJ (2000) Natural patterns of activity and long-term synaptic plasticity. Curr Opin Neurobiol 10:172-179.

Pavlides C, Greenstein YJ, Grudman M, Winson J (1988) Long-term potentiation in the dentate gyrus is induced preferentially on the positive phase of theta-rhythm. Brain Res 439:383-387.

Pittendrigh CS, Daan S (1976) A functional analysis of circadian pacemakers in nocturnal rodents. IV. Entrainment: pacemaker as clock. J Comp Physiol 106:291-331.

Renger JJ, Hartman KN, Tsuchimoto Y, Yokoi M, Nakanishi S, Hensch TK (2002) Experience-dependent plasticity without long-term depression by type 2 metabotropic glutamate receptors in developing visual cortex. Proc Natl Acad Sci USA 99:1041-1046.

Rozas C, Frank H, Heynen AJ, Morales B, Bear MF, Kirkwood A (2001) Developmental inhibitory gate controls the relay of activity to the superficial layers of the visual cortex. J Neurosci 21:6791-6801.

Sheng M, Cummings J, Roldan LA, Jan YN, Jan LY (1994) Changing subunit composition of heteromeric NMDA receptors during development of rat cortex. Nature 368:144-147.

Sherrington CS (1897) The central nervous system. In: A textbook of physiology, Part 3 (Foster M, ed). London: Macmillan.

Singer W (1995) Development and plasticity of cortical processing architectures. Science 270:758-759.

Steriade M, Timofeev I (2003) Neuronal plasticity in thalamocortical networks during sleep and waking oscillations. Neuron 37:563-576.

Steriade M, Deschênes M, Oakson G (1974) Inhibitory processes and interneuronal apparatus in motor cortex during sleep and waking. I. Background firing and responsiveness of pyramidal tract neurons and interneurons. J Neurophysiol 37:1065-1092. 
Steriade M, Contreras D, Curro Dossi R, Nunez A (1993) The slow $(<1 \mathrm{~Hz})$ oscillation in reticular thalamic and thalamocortical neurons: scenario of sleep rhythm generation in interacting thalamic and neocortical networks. J Neurosci 13:3284-3299.

Steriade M, Timofeev I, Grenier F, Dürmüller N (1998) Role of thalamic and cortical neurons in augmenting responses: dual intracellular recordings in vivo. J Neurosci 18:6425-6443.

Stickgold R, James L, Hobson JA (2000a) Visual discrimination learning requires sleep after training. Nat Neurosci 3:1237-1238.

Stickgold R, Whitbee D, Schirmer B, Patel V, Hobson JA (2000b) Visual discrimination improvement. A multi-step process occurring during sleep. J Cogn Neurosci 12:246-254.

Timofeev I, Contreras D, Steriade M (1996) Synaptic responsiveness of cortical and thalamic neurons during various phases of slow oscillation in cat. J Physiol (Lond) 494:265-278.

Timofeev I, Grenier F, Steriade M (2000) Impact of intrinsic properties and synaptic factors on the activity of neocortical networks in vivo. J Physiol (Paris) 94:343-355.

Tsanov M, Manahan-Vaughan D (2007) Intrinsic, light-independent and visual activity-dependent mechanisms cooperate in the shaping of the field response in rat visual cortex. J Neurosci 27:8422-8429.

Uzakov S, Frey JU, Korz V (2005) Reinforcement of rat hippocampal LTP by holeboard training. Learn Mem 12:165-171.

Wang H, Wagner JJ (1999) Priming-induced shift in synaptic plasticity in the rat hippocampus. J Neurophysiol 82:2024-2028.

Whitlock JR, Heynen AJ, Shuler MG, Bear MF (2006) Learning induces long-term potentiation in the hippocampus. Science 313 1093-1097.

Yoshimura Y, Ohmura T, Komatsu Y (2003) Two forms of synaptic plasticity with distinct dependence on age, experience, and NMDA receptor subtype in rat visual cortex. J Neurosci 23:6557-6566.

Zilles K (1985) The cortex of the rat. Berlin: Springer. 